\title{
Controlled Quantum Secure Direct Communication Protocol Based on Extended Three-particle GHZ State Decoy
}

\author{
Tao Fang \\ College of Fundamental Education Sichuan Normal University, Chengdu, China
}

\begin{abstract}
Extended three-particle GHZ state decoy is introduced in controlled quantum secure direct communication to improve eavesdropping detection probability and prevent correlation-elicitation (CE) attack. Each particle of extended three-particle GHZ state decoy is inserted into sending particles to detect eavesdroppers, which reaches $63 \%$ eavesdropping detection probability. And decoy particles prevent the receiver from obtaining the correct correlation between particle 1 and particle 2 before the sender coding on them, so that he can not get any secret information without the controller's permission. In the security analysis, the maximum amount of information that a qubit contains is obtained by introducing the entropy theory method, and two decoy strategies are compared quantitatively. If the eavesdroppers intend to eavesdrop on secret information, the per qubit detection rate of using only two particles of extended threeparticle GHZ state as decoy is 58\%; while the presented protocol using three particles of extended three-particle GHZ state as decoy reaches per qubit $63 \%$.
\end{abstract}

Keywords: CQSDC; extend three-particle GHZ state; decoy; eavesdropping detection; security

\section{Introduction}

One of the great advantages of quantum communication is the security. According to the principle of quantum mechanics, legal communicating parties can detect the existence of eavesdroppers efficiently, which ensures the security of communication[1].Quantum secure direct communication (QSDC) as a branch of quantum communication has attracted increasing attention[1-34]. The most important characteristic of QSDC is to transmit secret information in quantum communication channel by using the quantum no-cloning theorem, the uncertainty principle and the relevance and non-locality of entanglement particles [1]. "The efficient two-step quantum secure direct communication scheme " was proposed by Long Gui-Lu et al. in 2002, which is the first quantum secure direct communication protocol 1[2].After that, Deng Fu-Guo et al. put forward two-step quantum secure direct communication scheme based on dense coding, and discussed the standards of QSDC scheme[3]. Yan Feng-Li et al. proposed QSDC scheme based on quantum teleportation [4]. Yang et al. proposed the concept of generalized Bell state and unitary operations on the basis of logical qubits; based on it, a QSDC scheme was developed, which was immune to collective noise [17] In 2011, Lin et al. put forward a QSDC schema by using five-qubit entangled states.[20] In 2012, Huang et al. put forward a QSDC schema against collective noise with decoy states based on channel-encryption.[27]

Recently, as a distinctive concept, controlled quantum secure direct communication (CQSDC) [5-8] was pursued. Different from QSDC, CQSDC is used to communicate the secret message with the control of the controller, which is always applied to certain special cryptographic tasks. The receiver receives secret message from the sender through quantum channel, however he can not understand 
the message without the controller's permission. Wang Jian et al. introduced multiparty controlled quantum secure direct communication protocol (CQSDC); in this scheme, apart from sender and receiver, there is a controller; the sender sends secret information to receiver based on single-photon in quantum communication channel, while the receiver can not restore the information without controller's permission[5]. And then, the security of CQSDC scheme proposed by Wang Jian et al. was researched and analyzed by Wang Tian-Yin et al.; they put forward a new attack based on teleportation; by this attack, receiver could obtain secret message without controller's permission [6].In 2006, a new CQSDC scheme based on threeparticle Greenberger-Horne-Zeilinger (GHZ) state was put forward by Wang Jian et al. ,which achieves a high source capacity[7].Fei Gao et al. proposed correlationelicitation (CE) attack based on correlation extractability of GHZ state, and pointed that by this attack Wang Jian 's CQSDC scheme based on GHZ state would release 33.3\% secret message[8]. Recently, Li Jian et al. introduced decoy idea in QSDC to ensure security, extended three-particle GHZ state[9] was used as decoys, which brings per qubit $58 \%$ eavesdropping detection probability .

In this paper, decoy idea is introduced in CQSDC scheme based on extended three-particle GHZ state called DCQSDC scheme. Each particle of extended threeparticle GHZ state is used as decoy particle to detect eavesdropping. By using decoy designed in DCQSDC scheme, per qubit 63\% eavesdropping detection probability is reached. Decoy particles prevent receiver from obtaining the correct correlation between particle 1 and particle 2 before sender coding on them, so that he can not get any secret information without controller's permission. Therefore, CE attack is avoided efficiently. Comparing with protocol in [9], DCQSDC scheme improves eavesdropping detection probability. By using the DCQSDC protocol, the secret information can be securely transmitted to the receiver without any useful message being leaked out to the potential eavesdroppers.

\section{Description of DCQSDC Protocols}

Suppose that Bob is the controller, Alice is the sender, and Charlie is the receiver.

Step 1. The preparation stage. Bob and Alice prepare three-particle GHZ states sequences for transmitting secret information and extended three-particle GHZ states for detecting security of quantum communication channel.

S11). Bob prepares $\mathrm{N}$ ordered GHZ states for transmitting secret information, each of which is in the state:

$$
|\psi\rangle=\frac{1}{\sqrt{2}}(|000\rangle+|111\rangle)_{123}
$$

We denote the $\mathrm{N}$ three-particle GHZ states by $\left\{\left[p_{1}\left(S_{1}\right), p_{1}\left(S_{2}\right), p_{1}\left(S_{3}\right)\right]\right.$, $\left.\left[p_{2}\left(S_{1}\right), p_{2}\left(S_{2}\right), p_{2}\left(S_{3}\right)\right],\left[p_{3}\left(S_{1}\right), p_{3}\left(S_{2}\right), p_{3}\left(S_{3}\right)\right] \ldots\left[p_{N}\left(S_{1}\right), p_{N}\left(S_{2}\right), p_{N}\left(S_{3}\right)\right]\right\}$, wher e $p_{N}\left(S_{1}\right)$ denotes the first particle of the Nth GHZ state, $p_{N}\left(S_{2}\right)$ denotes the second particle of the Nth GHZ state, $p_{N}\left(S_{3}\right)$ denotes the third particle of the Nth GHZ state. Sequence composed by $\left\{p_{1}\left(S_{1}\right), p_{2}\left(S_{1}\right), \ldots p_{N}\left(S_{1}\right)\right\}$ is called $S_{1}$, sequence composed by $\left\{p_{1}\left(S_{2}\right), p_{2}\left(S_{2}\right), \ldots p_{N}\left(S_{2}\right)\right\}$ is called $S_{2}$, sequence composed by $\left\{p_{1}\left(S_{3}\right), p_{2}\left(S_{3}\right), \ldots p_{N}\left(S_{3}\right)\right\}$ is called $S_{3}$.

$\mathrm{S} 12)$. Bob prepares $\mathrm{M}$ ordered extended three-particle GHZ states as decoy for detecting eavesdropping when Bob sending particles to Alice, each of which is in the state 


$$
\left|\psi_{D}\right\rangle=\frac{1}{\sqrt{3}}(|000\rangle+|110\rangle+|111\rangle)_{123}
$$

Each extended three-particle GHZ state is called a decoy group, denoted by $D_{B A_{i}}, i=1,2 \cdots M$, and each particle of $D_{B A_{i}}$ is called decoy particle ,denoted by $D_{B A_{i 1}}, D_{B A_{i 2}}, D_{B A_{i 3}}$, sequence composed by $D_{B A_{i}}$ is called decoy sequence ,denoted by $D_{B A}$.

S13). According to method in S12) Bob prepares decoy sequence $D_{B C}$ for detecting eavesdropping when Bob sending particles to Charlie.

S14). According to method in S12) Alice prepares decoy sequence $D_{A C}$ for detecting eavesdropping when Alice sending particles to Charlie.

Step 2. The stage of sending messages and detecting eavesdropping.

S21). Bob inserts decoy particles of each group in $D_{B A}$ to $S_{1}$ randomly, composing new sequence $S_{1}^{\prime}$. For example, Bob can insert $D_{B A}$ to $S_{1}$ like that:

$$
\begin{aligned}
& \quad S_{1} \text { 序列 } \\
& \quad p_{1}\left(S_{1}\right), p_{2}\left(S_{1}\right), p_{3}\left(S_{1}\right), p_{4}\left(S_{1}\right), p_{5}\left(S_{1}\right), \cdots p_{N}\left(S_{1}\right) \\
& S_{1}^{\prime} \text { 序列 } \\
& p_{1}\left(S_{1}\right), D_{B A_{1}}, p_{2}\left(S_{1}\right), D_{B A_{12}}, p_{3}\left(S_{1}\right), D_{B A_{13}}, p_{4}\left(S_{1}\right), D_{B A_{21}}, \cdots p_{N}\left(S_{1}\right)
\end{aligned}
$$

S22). Bob performs Pauli $\left\{I, \sigma_{z}, \sigma_{x}, \sigma_{i y}\right\}$ operation randomly on each particle in $S_{2}$, generating sequence $S_{2}^{\prime}$. Bob sends $S_{1}^{\prime}$ and $S_{2}^{\prime}$ to Alice. Bob tells Alice the positions of decoy particles through classical communication channel.

S23). Alice extracts decoy particles according to the positions Bob told her after she receives the particles sequence. Alice reassembles the $D_{B A_{i}}$ groups by the extracted decoy particles. Alice performs extended three-particle GHZ measurement on each $D_{B A_{i}}$ group. If there is no eavesdropper, every result should be in the extended three-particle GHZ state .Alice discards decoy particles and recovers $S_{1}$ sequence, and then go to the next step. If there is eavesdropper, go back to step1.

S24). Bob inserts decoy particles of each group in $D_{B C}$ to $S_{3}$ randomly, composing new sequence $S_{3}^{\prime}$, and sends it to Charlie. The method to insert decoy particles is similar with that in S21). Charlie detects eavesdropping by using method similar with that in S23). If there is no eavesdropper, Bob discards decoy particles and recovers $S_{3}$ sequence, then go to the next step. If there is eavesdropper, go back to step1.

S25). Alice encrypts her secure message by randomly performing eight Paulipairs operation on $S_{1}$ and $S_{2}^{\prime}$ sequence, and forms coding sequence $C_{1}$ and $C_{2}^{\prime}$.The Pauli-pairs are:

$$
\begin{aligned}
& \left\{U_{1}=\sigma_{z} \otimes \sigma_{z}, U_{2}=I \otimes \sigma_{z}, U_{3}=i \sigma_{y} \otimes \sigma_{z}, U_{4}=\sigma_{x} \otimes \sigma_{z},\right. \\
& \left.U_{5}=I \otimes \sigma_{x}, U_{6}=\sigma_{x} \otimes \sigma_{z}, U_{7}=\sigma_{x} \otimes \sigma_{x}, U_{4}=i \sigma_{y} \otimes \sigma_{x}\right\}
\end{aligned}
$$

S26). Alice inserts decoy particles of each group in $D_{A C}$ to $C_{1}$ and $C_{2}^{\prime}$ randomly, and sends them to Charlie. The method to insert decoy particles is similar with that in S21).Charlie detects eavesdropping by using method similar with that in S23). If 
there is no eavesdropper, Charlie discards decoy particles and recovers $C_{1}$ and $C_{2}{ }^{\prime}$, then go to the next step. If there is eavesdropper, go back to step1.

S27). Bob tells Charlie the Pauli operations that he performed on $S_{2}$ through classical channel. Charlie performs corresponding Pauli operations on $C_{2}^{\prime}$, and obtained $C_{2}$.Charlie performs GHZ measurement on $C_{1}, C_{2}$ and $S_{3}$, then gets the information Alice sent to him.

\section{Security Analysis}

\subsection{Analysis of Eavesdropping Detection Probability}

Suppose that Eve is eavesdropping When Bob sends particles sequence mixed with decoy particles to Alice, because Eve does not know which particle is decoy particle, he can only performs the same attack operation on all particles. On assumption that the attack operation Eve performs on particles is $E$, after performing the attack operation $E$, the original $|0\rangle$ state becomes $\left|0^{\prime}\right\rangle=$ $E \otimes|0 x\rangle=\alpha\left|0 x_{0}\right\rangle+\beta\left|1 x_{1}\right\rangle$; and the original $|1\rangle$ state becomes $\left|1^{\prime}\right\rangle=E \otimes|1 x\rangle=m\left|0 y_{0}\right\rangle+n\left|1 y_{1}\right\rangle ;|\alpha|^{2}+|\beta|^{2}=1, \quad|m|^{2}+|n|^{2}=1$.

After being attacked by Eve, the state of the composed system becomes:

$$
\begin{aligned}
|\psi\rangle_{E v e}= & E \otimes E \otimes E\left[\frac{1}{\sqrt{3}}(|0 x 0 x 0 x\rangle+|1 x 1 x 0 x\rangle+|1 x 1 x 1 x\rangle)\right] \\
= & \frac{1}{\sqrt{3}}\left[\left(\alpha\left|0 x_{0}\right\rangle+\beta\left|1 x_{1}\right\rangle\right) \otimes\left(\alpha\left|0 x_{0}\right\rangle+\beta\left|1 x_{1}\right\rangle\right) \otimes\left(\alpha\left|0 x_{0}\right\rangle+\beta\left|1 x_{1}\right\rangle\right)\right. \\
& +\left(m\left|0 y_{0}\right\rangle+n\left|1 y_{1}\right\rangle\right) \otimes\left(m\left|0 y_{0}\right\rangle+n\left|1 y_{1}\right\rangle\right) \otimes\left(\alpha\left|0 x_{0}\right\rangle+\beta\left|1 x_{1}\right\rangle\right) \\
& \left.+\left(m\left|0 y_{0}\right\rangle+n\left|1 y_{1}\right\rangle\right) \otimes\left(m\left|0 y_{0}\right\rangle+n\left|1 y_{1}\right\rangle\right) \otimes\left(m\left|0 y_{0}\right\rangle+n\left|1 y_{1}\right\rangle\right)\right] \\
= & \frac{1}{\sqrt{3}}\left(\alpha^{3}\left|0 x_{0} 0 x_{0} 0 x_{0}\right\rangle+\alpha^{2} \beta\left|0 x_{0} 0 x_{0} 1 x_{1}\right\rangle+\alpha \beta \alpha\left|0 x_{0} 1 x_{1} 0 x_{0}\right\rangle+\alpha \beta^{2}\left|0 x_{0} 1 x_{1} 1 x_{1}\right\rangle\right. \\
& +\beta \alpha^{2}\left|1 x_{1} 0 x_{0} 0 x_{0}\right\rangle+\beta \alpha \beta\left|1 x_{1} 0 x_{0} 1 x_{1}\right\rangle+\beta^{2} \alpha\left|1 x_{1} 1 x_{1} 0 x_{0}\right\rangle+\beta^{3}\left|1 x_{1} 1 x_{1} 1 x_{1}\right\rangle \\
+ & m m \alpha\left|0 y_{0} 0 y_{0} 0 x_{0}\right\rangle+m m \beta\left|0 y_{0} 0 y_{0} 1 x_{1}\right\rangle+m n \alpha\left|0 y_{0} 1 y_{1} 0 x_{0}\right\rangle+m n \beta\left|0 y_{0} 1 y_{1} 1 x_{1}\right\rangle \\
+ & n m \alpha\left|1 y_{1} 0 y_{0} 0 x_{0}\right\rangle+n m \beta\left|1 y_{1} 0 y_{0} 1 x_{1}\right\rangle+n n \alpha\left|1 y_{1} 1 y_{1} 0 x_{0}\right\rangle+n n \beta\left|1 y_{1} 1 y_{1} 1 x_{1}\right\rangle \\
+ & m^{3}\left|0 y_{0} 0 y_{0} 0 y_{0}\right\rangle+m^{2} n\left|0 y_{0} 0 y_{0} 1 y_{1}\right\rangle+m n m\left|0 y_{0} 1 y_{1} 0 y_{0}\right\rangle+m n^{2}\left|0 y_{0} 1 y_{1} 1 y_{1}\right\rangle \\
+ & \left.n m^{2}\left|1 y_{1} 0 y_{0} 0 y_{0}\right\rangle+n m n\left|1 y_{1} 0 y_{0} 1 y_{1}\right\rangle+n^{2} m\left|1 y_{1} 1 y_{1} 0 y_{0}\right\rangle+n^{3}\left|1 y_{1} 1 y_{1} 1 y_{1}\right\rangle\right)
\end{aligned}
$$

If the extended three-particle GHZ measurement is performed on the decoy particles, the probability that Alice gets correct measurement results is just the probability without eavesdroppers, which can be calculated as:

$p\left(\left|\psi_{E}\right\rangle\right)=\frac{1}{3}\left(\left|\alpha^{3}\right|^{2}+\left|m^{2} \alpha\right|^{2}+\left|m^{3}\right|^{2}+\left|\beta^{2} \alpha\right|^{2}+\left|n^{2} \alpha\right|^{2}+\left|n^{2} m\right|^{2}+\left|\beta^{3}\right|^{2}+\left|n^{2} \beta\right|^{2}+\left|n^{3}\right|^{2}\right)$

Suppose $|\alpha|^{2}=a,|\beta|^{2}=b,|m|^{2}=s,|n|^{2}=t$, because $a+b=1, s+t=1$, the lower bound of the probability that every qubit is eavesdropped on and is detected is:

$p\left(\left|\psi_{E}\right\rangle\right)=\frac{1}{3}\left(a^{3}+a(1-t)^{2}+(1-t)^{3}+(1-a)^{2} a+t^{2} a+t^{2}(1-t)+(1-a)^{3}+t^{2}(1-a)+t^{3}\right)$ 


$$
=\frac{1}{3}\left(a^{3}-a-2 a t+a t^{2}+2-3 t+5 t^{2}+a^{2}-t^{3}\right)
$$

It can be considered that the Shannon entropy of a binary channel is equal to the maximum amount of information that a qubit contains. If Bob sends a particle in state $|0\rangle$ to Alice, then the maximum amount of information containing in the $|0\rangle$ state qubit is:

$$
I_{|0\rangle}=-a \log _{2} a-(1-a) \log _{2}(1-a)=H(a)
$$

If Bob sends a particle in state $|1\rangle$ to Alice, then the maximum amount of information containing in the $|1\rangle$ state qubit is:

$$
I_{|1\rangle}=-t \log _{2} t-(1-t) \log _{2}(1-t)=H(t)
$$

Because the particle that Bob sends to Alice is considered in either of the states $|0\rangle$ or $|1\rangle$ with equal probability $\mathrm{p}=0.5$, we can get $a=t$. So for a qubit, the total amount of information that will be eavesdropped by Eve is:

$$
I=I_{|0\rangle}+I_{|1\rangle}=\frac{1}{2}[H(a)+H(t)]=H(a)
$$

Because $a=t$, then $p\left(\left|\psi_{E}\right\rangle\right)==\frac{1}{3}\left(a^{3}+4 a^{2}-4 a+2\right)$

$$
d_{\text {low }}=1-p\left(\left|\psi_{E}\right\rangle\right)=1-\frac{1}{3}\left(a^{3}+4 a^{2}-4 a+2\right)
$$

When $I=1, d_{\text {low }}=0.63$. That is, the detection probability is more than $63 \%$, when Eve intends to eavesdrop on the information contained in a qubit.

In lecture [9] only two particles of extended three-particle GHZ state are used as decoy, which brought per qubit $58 \%$ eavesdropping detection probability. However in this DCQSDC scheme, three particles of extended three-particle GHZ state are used to design decoy, which reaches per qubit 63\% eavesdropping detection probability.

\subsection{CE Attack}

In lecture [8], CQSDC scheme based on GHZ state is pointed out existing CE attack. That is, if Charlie can obtain the correlation between particle 1 and 2 before Alice's coding operations, he can get 33.3\% information about this secret without Bob's permission. In this DCQSDC scheme, Bob inserts decoy particles to sequence $S_{1}$ randomly before sending it to Alice. Because Charlie does not know which particle is the decoy particle, he can not get the correlation between particle 1 and 2 before Alice's coding operations, he consequently can not obtain the secret information without Bob's permission. Furthermore, each particle's sending accompanied by decoy particles, and the eavesdropping detection probability of per qubit is more than $63 \%$, so Charlie will be easily found if he eavesdrops.

\section{Conclusions}

In the DCQSDC protocol presented in this paper, each particle of extended threeparticle GHZ state is used as decoy particle to detect eavesdroppers, which reaches per qubit $63 \%$ eavesdropping detection probability. At the same time randomly introducing decoy particle in CQSDC scheme prevents the secret information from CE attack efficiently. Comparing with protocol in [9], DCQSDC scheme improves eavesdropping detection probability. By using the DCQSDC protocol, the secret 
information can be securely transmitted to the receiver without any useful message being leaked out to the potential eavesdroppers.

\section{Acknowledgements}

This work was supported by Sichuan Department of Education (Grant No.13ZB0152).

\section{References}

[1] G. L. LONG, C. WANG, Y. S. LI, et al. Quantum secure direct communication[J]. SCIENTIA SINICA Phys, Mech \& Astron, 41(4):332-342(2011)

[2] G. L. LONG, X. S. LIU. Theoretically efficient high-capacity quantum-key-distribution scheme[J]. Phys Rev A, 65: 032302(2002)

[3] F. G. DENG, G. L. LONG, X. S. LIU. Two-step quantum direct communication protocol using the Einstein-Podolsky-Rosen pair block. Phys Rev A, 68(4): 042317(2003)

[4] F. L. YAN, X. Q. ZHANG. A scheme for secure direct communication using EPR pairs and teleportation. Eur Phys J B, 41: 75-78(2004)

[5] J. WANG, H. Q. CHEN, Q. ZHANG, et al. Multiparty controlled quantum secure direct communication protocol[J].ACTA PHYSICA SINICA, 56(2):673-676(2007)

[6] T. Y. WANG, S. J. QIN, Q. Y. WEN, et al. Analysis and improvement of multiparty controlled quantum secure direct communication protocol[J].ACTA PHYSICA SINICA, 57(12):7452-7457(2008)

[7] J. WANG, Q. ZHANG, C. J. TANG. Opt. Commun. 266(2006)

[8] G. FEI, S. J. QIN, Q. Y. WEN, et al. Cryptanalysis of multiparty controlled quantum secure direct communication using Greenberger-Horne-Zeilinger state[J]. Optics Communications, 192-195(2010)

[9] J. LI, X. J. GUO, D. J. SONG, et al. Improved Quantum "Ping-Pong" Protocol Based on Extended Three-Particle GHZ State[J].China Communications, 1:111-115(2012)

[10] G. L. LONG, C. WANG, F. G.DENG, et al. Quantum Direct Communication, Advances in Lasers and Electro Optics. Nelson Costa, Adolfo Cartaxo, Eds. INTECH(2010)

[11] G. L. LONG, F. G.DENG,C. WANG, et al. Quantum secure direct communication and deterministic secure quantum communication. Front Phys China, 2(3): 251-272 (2007)

[12] B. GU, X. PEI S, B. SONG, et al. Deterministic secure quantum communication over a collective-noise channel. Sci China Ser G-Phys Mech Astron, 52(12): 1913-1918(2009)

[13] Y. G. YANG, Q. Y. WEN, F. C. ZHU. An efficient quantum secure direct communication scheme with authentication. Chin Phys, 16(7):1838-1842(2007)

[14] F. GAO, Q. Y .WEN, F. C. ZHU. Teleportation attack on the QSDC protocol with a random basis and order. Chin Phys B, 17(9):3189-3193(2008)

[15] J. LI, D J. SONG, X. J. GUO, et al. A quantum secure direct communication protocol based on a fiveparticle cluster state and classical XOR operation[J].Chinese Physics C, 36(1):31-36(2012)

[16] F. Gao, S. J. Qin, Q. Y. Wen, et al. Cryptanalysis of multiparty controlled quantum secure direct communication using Greenberger-Horne-Zeilinger state. Opt. Commun., 283:192-195(2010)

[17] J. Yang, C. Wang, R. Zhang. Faithful quantum secure direct communication protocol against collective noise. Chin. Phys. B, 19: 110311(2010)

[18] F. Z. Guo, S. J. Qin, Q. Y. Wen ,et al. Cryptanalysis and Improvement of Two GHZ-State-Based QSDC Protocols. Chin. Phys. Lett., 27: 090307(2010)

[19] F. Gao, S. J. Qin, F. Z. Guo, et al. Cryptanalysis of Quantum Secure Direct Communication and Authentication Scheme via Bell States. Chin. Phys. Lett., 28: 020303(2011)

[20] S. Lin, F. Gao, X. F. Liu. Quantum Secure Direct Communication with Five-Qubit Entangled State. Chin. Phys. Lett., 28: 030302(2011)

[21] T. J. Wang, T. Li, F. F. Du, et al. High-Capacity Quantum Secure Direct Communication Based on Quantum Hyperdense Coding with Hyperentanglement. Chin. Phys. Lett., 28: 040305(2011)

[22] B. Gu, Y. G .Huang, X. Fang, et al. Bidirectional Quantum Secure Direct Communication Network Protocol with Hyperentanglement. Commun. Theor. Phys., 56: 659-663(2011)

[23] B. Gu, Y. G. Huang, X. Fang, et al. A two-step quantum secure direct communication protocol with hyperentanglement. Chin. Phys. B, 20: 100309(2011)

[24] B. Gu, C. Y. Zhang, G. S. Cheng, et al. Robust quantum secure direct communication with a quantum one-time pad over a collective-noise channel. Sci. China.Phys. Mech. Astron., 54:942-947(2011)

[25] J. Shi, Y. X. Gong, P. Xu, et al. Quantum Secure Direct Communication by Using Three-Dimensional Hyperentanglement. Commun. Theor. Phys., 56: 831(2011)

[26] G. Gao, M. Fang, L. P. Wang, et al. Individual Attack on the Improved Multiparty Quantum Secret Sharing Protocol. Int. J. Theor. Phys., 50: 1726-1730(2011)

[27] W. Huang, Q. Y. Wen, H. Y. Jia, et al. Fault tolerant quantum secure direct communication with quantum encryption against collective noise. Chin. Phys. B, 21: 100308(2012)

[28] B. C. Ren, H. R. Wei, M. Hua, et al. Photonic spatial Bell-state analysis for robust quantum secure direct communication using quantum dot-cavity systems. Eur. Phys. J. D, 67:30(2013) 
[29] C. W. Yang, C. W. Tsai, T. Hwang. Fault tolerant two-step quantum secure direct communication protocol against collective noises, SCIENCE CHINA Physics, Mechanics \& Astronomy, 54(3): 496501(2011)

[30] C.W.Yang, T. Hwang. Fault tolerant authenticated quantum direct communication immune to collective noises, Quantum Inf Process, 12(11): 3495-3509(2013)

[31] H.C Niu, B. C. Ren, T. J. Wang. Faithful Entanglement Sharing for Quantum Communication Against Collective Noise, Int J Theor Phys, 51(8): 2346-2352(2012)

[32] B. Gu, Y.G. Huang, X. Fang, et al. Robust Quantum Secure Communication with Spatial Quantum States of Single Photons, Int J Theor Phys, 52(12): 4461-4469(2013)

[33] T. J. Wang, T. Li, F. F. Du, et al. High-capacity quantum secure direct communication based on quantum hyperdense coding with hyperentanglement, Chin Phys Lett, 28(4): 040305(2011)

[34] R. T. Zhao, Q. Guo, L. Chen. Quantum superdense coding based on hyperentanglement, Chin Phys B, 21(8):080303(2012)

\section{Author}

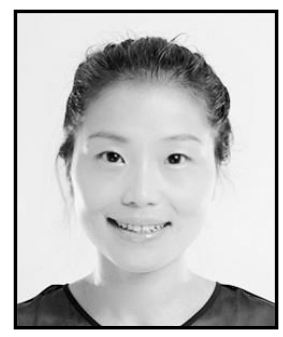

Tao Fang, received the M.S. degree from the Sichuan Normal University. Her main research interests include the network secu rity protocol, privacy protection. 
International Journal of Security and Its Applications

Vol.9, No.10 (2015) 